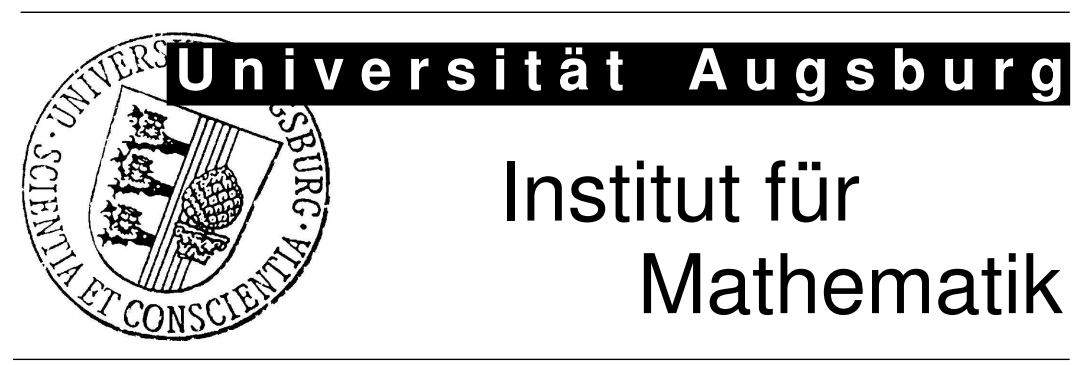

Christoph Kawan, Torben Stender

\title{
Lipschitz Conjugacy of Linear Flows
}

Preprint Nr. 14/2008 - 28. März 2008 


\section{Impressum:}

Herausgeber:

Institut für Mathematik

Universität Augsburg

86135 Augsburg

http://www . math. uni-augsburg.de/forschung/preprint/

ViSdP:

Torben Stender Institut für Mathematik

Universität Augsburg

86135 Augsburg

Preprint: Sämtliche Rechte verbleiben den Autoren (C) 2008 


\title{
Lipschitz Conjugacy of Linear Flows
}

\author{
C. Kawan and T. Stender
}

\begin{abstract}
In this paper we characterize Lipschitz conjugacy of linear flows on $\mathbb{R}^{d}$ algebraically. We show that two hyperbolic linear flows are Lipschitz conjugate if and only if the Jordan forms of the system matrices are the same except for the simple Jordan blocks where the imaginary parts of the eigenvalues may differ. Using a well-known result of Kuiper we obtain a characterization of Lipschitz conjugacy for arbitrary linear flows.
\end{abstract}

\section{Introduction}

The flow induced by the linear autonomous differential equation $\dot{x}=A x$ is given by $(t, x) \mapsto e^{A t} x$. Two linear flows $e^{A t} x$ and $e^{B t} x$ are said to be topologically conjugate if there exists a homeomorphism $h: \mathbb{R}^{d} \rightarrow \mathbb{R}^{d}$ such that $h\left(e^{A t} x\right)=e^{B t} h(x)$ for all $x \in \mathbb{R}^{d}$ and $t \in \mathbb{R}$. In the hyperbolic case, i.e. if $A$ and $B$ have no eigenvalues with real part zero, the flows $e^{A t} x$ and $e^{B t} x$ are topologically conjugate if and only if the dimensions of the stable eigenspaces coincide (cf. [1, Theorem 7.1., p. 113]). In the case of $C^{1}$-conjugacy, which means topological conjugacy by a $C^{1}$-diffeomorphism, we obtain a quite different result: Two linear flows $e^{A t} x$ and $e^{B t} x$ (not necessarily hyperbolic) are $C^{1}$-conjugate if and only if they are linearly conjugate. This can be proved very easily by differentiating the conjugacy identity. Hence there is a big gap between topological and differentiable conjugacy. A property of maps that lies between continuity and differentiability is Lipschitz continuity. In the present paper we study conjugacy of linear flows by bi-Lipschitz homeomorphisms. A famous theorem of Rademacher says that a Lipschitz continuous map is differentiable Lebesgue almost everywhere. Thus Lipschitz continuity is very close to differentiability and as we show in this paper, Lipschitz conjugacy is very close to $C^{1}$-conjugacy and therefore to linear conjugacy. Our main theorem states that two hyperbolic flows $e^{A t} x$ and $e^{B t} x$ are Lipschitz conjugate if and only if the real Jordan forms of $A$ and $B$ coincide except for the simple Jordan blocks, where the imaginary parts of the eigenvalues may differ. Using a result of Kuiper (cp. [2, 1 1] or [3, Theorem B'] for a different formulation) we obtain that arbitrary linear flows are Lipschitz conjugate if and only if their hyperbolic parts are Lipschitz conjugate and their non-hyperbolic parts are linearly conjugate.

This paper is organized as follows: In section 3 we repeat some facts about Lipschitz continuous maps including the theorem of Rademacher, and we give a sufficient condition for a map to satisfy a global Lipschitz condition (Lemma 3.2). In section 4 the notion of kinematic similarity of (nonautonomous) linear differential equations is introduced and characterized algebraically in the autonomous case. Kinematical similarity is a generalization of linear conjugacy. Unlike in the case of a linear transformation, a kinematical similarity transformation may vary in time but has to be bounded together with its inverse. This notion was first introduced by Perron in his stability theory (see [4]). In [5] Markus characterizes kinematic similarity within the set of systems that are kinematically similar to an autonomous system by giving a complete set of invariants. A consequence of his result is that two autonomous systems 
are kinematically similar if and only if the complex Jordan forms of the system matrices coincide after dropping the imaginary parts of the eigenvalues. We state this fact in Proposition 4.2 and give a proof which is partially based on the results of Markus. In section 5 we state and prove our main theorem, Theorem 5.6. To this end, we first have to prove some auxiliary results. In particular, using Lemma 3.2 we verify the mentionable fact that two hyperbolic linear flows are Lipschitz conjugate if and only if they are conjugate by a homeomorphism which, together with its inverse, satisfies a global Lipschitz condition (Corollary 5.4). Using this result we can show that Lipschitz conjugacy implies kinematic similarity of both the given systems and of two associated systems. The algebraical characterization of kinematic similarity provided in section 4 then yields an algebraical characterization of Lipschitz conjugacy.

\section{Preliminaries}

By $\mathbb{R}$ we denote the reals, by $\mathbb{R}^{d}$ the $d$-dimensional Euclidean space. $\mathbb{R}^{d \times d}$ is set of $d \times d$ matrices with entries in $\mathbb{R}$ and $\operatorname{Gl}(d, \mathbb{R}) \subset \mathbb{R}^{d \times d}$ the general linear group. By $\langle\cdot, \cdot\rangle$ we denote the Euclidean scalar product on $\mathbb{R}^{d}$ and by $\|x\|=\langle x, x\rangle^{\frac{1}{2}}$ the Euclidean norm of $x \in \mathbb{R}^{d}$. We write $\|A\|$ for the operator norm of the matrix $A \in \mathbb{R}^{d \times d}$ induced by the Euclidean norm, i.e. $\|A\|=\sup _{\|v\|=1}\|A v\| . I=I_{d} \in \mathbb{R}^{d \times d}$ is the identity matrix. A diagonal block matrix with blocks $A_{1}, \ldots, A_{r}, A_{i} \in \mathbb{R}^{r_{i} \times r_{i}}$, is denoted by $\operatorname{diag}\left(A_{1}, \ldots, A_{r}\right)$. The Lyapunov space of $A \in \mathbb{R}^{d \times d}$ corresponding to the real number $\lambda$ is the sum of all real generalized eigenspaces of $A$ corresponding to eigenvalues with real part $\lambda$. By $\lambda^{d}$ we denote the $d$-dimensional Lebesgue measure on $\mathbb{R}^{d}$. For any set $S \subset \mathbb{R}^{d}$ we write $\chi_{S}$ for the characteristic function of $S$, i.e. $\chi_{S}(x)=1$ if $x \in S$ and $\chi_{S}(x)=0$ otherwise. If $f: \mathbb{R}^{d} \rightarrow \mathbb{R}^{d}$ is a map which is differentiable at $\xi \in \mathbb{R}^{d}$, we denote the corresponding Jacobi matrix by $D h_{\xi}=D h(\xi)$.

In this paper we use the the notion real Jordan form in a somewhat unconventional manner. Usually, for a matrix $A \in \mathbb{R}^{d \times d}$ one obtains the real Jordan form from the complex Jordan form by combining $r \times r$ Jordan blocks corresponding to a complex pair $\lambda \pm i \mu$ of eigenvalues to one $2 r \times 2 r$ block of the form

$$
\left[\begin{array}{ccccccc}
\lambda & \mu & 1 & & & & \\
-\mu & \lambda & & 1 & & & \\
& & \cdot & & \cdot & & \\
& & & \cdot & & 1 & \\
& & & & \cdot & & 1 \\
& & & & & \lambda & \mu \\
& & & & & -\mu & \lambda
\end{array}\right]
$$

This is done for all nonreal eigenvalues $\lambda+i \mu$ of $A$. In addition to this we also combine such blocks if $\lambda+i \mu$ is real, i.e. if $\mu=0$. This means, if $\lambda$ is a real eigenvalue of $A$ and the $r \times r$ Jordan block

$$
\left[\begin{array}{lllll}
\lambda & 1 & & & \\
& \cdot & \cdot & & \\
& & \cdot & \cdot & \\
& & & \cdot & 1 \\
& & & & \lambda
\end{array}\right]
$$


appears twice in the complex Jordan form, we combine the two blocks to obtain the $2 r \times 2 r$ block

$$
\left[\begin{array}{lllllll}
\lambda & & 1 & & & & \\
& \lambda & & 1 & & & \\
& & \cdot & & \cdot & & \\
& & & \cdot & & 1 & \\
& & & & \cdot & & 1 \\
& & & & & \lambda & \\
& & & & & & \lambda
\end{array}\right] .
$$

This has the following advantage: If $A$ and $B$ are Lyapunov blocks, i.e. if all eigenvalues have the same real part, and if the nilpotent parts of the complex Jordan forms coincide, then also the nilpotent parts of the real Jordan forms do (modulo rearranging the blocks).

For any matrix $A \in \mathbb{R}^{d \times d}$ the flow induced by the differential equation $\dot{x}=A x$ is briefly denoted by $e^{A t} x$. For an eigenvalue $\lambda$ of $A$ we denote the corresponding real generalized eigenspace by $E_{\lambda}^{g}(A)$. We define the linear subspaces $\mathbb{E}^{\sigma}, \sigma=s, u, c$, by

$$
\begin{aligned}
& \mathbb{E}^{s}:=\mathbb{E}^{s}(A):=\bigoplus_{\operatorname{Re}(\lambda)<0} E_{\lambda}^{g}(A) \quad \text { (stable eigenspace), } \\
& \mathbb{E}^{u}:=\mathbb{E}^{u}(A):=\bigoplus_{\operatorname{Re}(\lambda)>0} E_{\lambda}^{g}(A) \quad \text { (unstable eigenspace), } \\
& \mathbb{E}^{c}:=\mathbb{E}^{c}(A):=\bigoplus_{\operatorname{Re}(\lambda)=0} E_{\lambda}^{g}(A) \quad \text { (center eigenspace). }
\end{aligned}
$$

The subspaces $\mathbb{E}^{\sigma}$ are invariant under the flow $e^{A t} x$, i.e. $e^{A t} \mathbb{E}^{\sigma}=\mathbb{E}^{\sigma}$ for all $t \in \mathbb{R}$, and they can be characterized dynamically in the following way (see [1, Theorem 6.1., p. 111]):

$$
\begin{aligned}
& \mathbb{E}^{s}=\left\{v \in \mathbb{R}^{d} \mid \exists a>0, C \geq 1:\left\|e^{A t} v\right\| \leq C e^{-a t}\|v\| \text { for } t \geq 0\right\}, \\
& \mathbb{E}^{u}=\left\{v \in \mathbb{R}^{d} \mid \exists a>0, C \geq 1:\left\|e^{A t} v\right\| \leq C e^{-a|t|}\|v\| \text { for } t \leq 0\right\}, \\
& \mathbb{E}^{c}=\left\{v \in \mathbb{R}^{d} \mid \forall a>0:\left\|e^{A t} v\right\| e^{-a|t|} \rightarrow 0 \text { as } t \rightarrow \pm \infty\right\}
\end{aligned}
$$

Hence solutions starting in $\mathbb{E}^{s}$ converge with exponential speed to zero in forward time, and solutions starting in $\mathbb{E}^{u}$ show the same behaviour in backward time. Solutions starting in $\mathbb{E}^{c}$ grow at most subexponentially both in forward and in backward time. If $\mathbb{E}^{c}=\{0\}$, or equivalently, if $A$ has no eigenvalues with real part zero, then the differential equation $\dot{x}=A x$ and the flow $e^{A t} x$ are called hyperbolic.

\section{Lipschitz continuous maps}

In this section we repeat some facts on Lipschitz continuous maps and prove a technical lemma which yields a sufficient condition for a map to satisfy a global Lipschitz condition.

A map $f: X \rightarrow Y$ between metric spaces $\left(X, d_{X}\right)$ and $\left(Y, d_{Y}\right)$ is said to be Lipschitz continuous if for every point $x \in X$ there exists a neighborhood $U$ of $x$ and a Lipschitz constant $L \geq 0$ for $\left.f\right|_{U}$, i.e.

$$
d_{Y}\left(f\left(x_{1}\right), f\left(x_{2}\right)\right) \leq L d_{X}\left(x_{1}, x_{2}\right) \text { for all } x_{1}, x_{2} \in U .
$$

Every Lipschitz continuous map $f: X \rightarrow Y$ is continuous, and if the metric spaces $X$ and $Y$ are Riemannian manifolds, then every $C^{1}$-map $f: X \rightarrow Y$ is Lipschitz continuous. If $X=Y=\mathbb{R}^{d}$, then in particular every linear map $f: X \rightarrow Y$ is Lipschitz continuous with respect to any 
metric induced by a norm. If a map $f: X \rightarrow Y$ is invertible and both $f$ and $f^{-1}$ are Lipschitz continuous, we call $f$ a bi-Lipschitz homeomorphism. If $f: X \rightarrow Y$ satisfies $d_{Y}\left(f\left(x_{1}\right), f\left(x_{2}\right)\right) \leq$ $L d_{X}\left(x_{1}, x_{2}\right)$ for all $x_{1}, x_{2} \in X$ with a constant $L \geq 0$, we say that $f$ satisfies a global Lipschitz condition, or that $f$ is globally Lipschitz continuous. The following theorem of Rademacher (see also [6, Th. 5.5.7, p. 196]) reveals that Lipschitz continuity is much stronger than continuity and is indeed almost differentiability.

Theorem 3.1. Let $M$ and $N$ be Riemannian manifolds and $f: M \rightarrow N$ a Lipschitz continuous map. Then $f$ is differentiable almost everywhere, i.e. the set of points $x \in M$, where $f$ is not differentiable, has Lebesgue measure zero.

For every continuous map $f: M \rightarrow N$ between manifolds $M$ and $N$ we introduce the set

$$
\Delta(f):=\left\{x \in M \mid D f_{x} \text { exists }\right\} .
$$

Theorem 3.1 says that the set $M \backslash \Delta(f)$ has Lebesgue measure zero if $f$ is Lipschitz continuous.

For $x, y \in \mathbb{R}^{d}$ we define the straight line segment

$$
[x, y]:=\{(1-t) x+t y \mid t \in[0,1]\} .
$$

Moreover, we define $(x, y):=[x, y] \backslash\{x, y\}$.

Lemma 3.2. Let $f: \mathbb{R}^{d} \rightarrow \mathbb{R}^{d}$ be a Lipschitz continuous map such that there exists a constant $L \geq 0$ with

$$
\left\|D f_{x}\right\| \leq L \text { for all } x \in \Delta(f) .
$$

Then $f$ satisfies a global Lipschitz condition with Lipschitz constant L.

Proof. $\quad$ By Theorem 3.1 the set $\mathbb{R}^{d} \backslash \Delta(f)$ has Lebesgue measure zero. By a well-known result in measure theory there exists a set $C \subset \mathbb{R}^{d}$ which is the countable union of closed sets (a socalled $F_{\sigma}$-set) such that $C \subset \Delta(f)$ and $\lambda^{d}(\Delta(f) \backslash C)=0$ (see e.g. [7, Lem. 1.5.3., p. 37]). Since $\mathbb{R}^{d} \backslash C$ is the disjoint union of $\mathbb{R}^{d} \backslash \Delta(f)$ and $\Delta(f) \backslash C$ this implies

$$
\lambda^{d}\left(\mathbb{R}^{d} \backslash C\right)=0 .
$$

As the countable union of closed sets $C$ is a Borel set. Consequently, the intersection of $C$ with every line segment $[x, y]$ is a Borel set in $[x, y]$, and thus, Lebesgue measurable with respect to the one-dimensional Lebesgue measure on $[x, y]$. We fix a point $x \in \mathbb{R}^{d}$ and define the set

$$
C_{x}:=\left\{y \in \mathbb{R}^{d} \backslash\{x\} \mid[x, y] \cap C \text { has full } \lambda^{1} \text {-measure }\right\},
$$

We want to show that $C_{x}$ is dense in $\mathbb{R}^{d}$. To this end, we assume that the converse holds, i.e. $\mathbb{R}^{d} \backslash \bar{C}_{x} \neq \emptyset$. Then we find $z \in \mathbb{R}^{d}$ and $r>0$ such that the open ball $U:=\left\{x \in \mathbb{R}^{d} \mid \| x-\right.$ $z \|<r\}$ is contained in $\mathbb{R}^{d} \backslash \bar{C}_{x}$. Consider the $(d-1)$-dimensional hyperplane $S$ through $z$ which is orthogonal to $z-x$,

$$
S=\{z+w \mid\langle w, z-x\rangle=0\} .
$$

The set $S \cap U$ is a $(d-1)$-dimensional ball in $S$ and hence contains a $(d-1)$-dimensional open box $Q$, i.e. a set which is isometric to the Cartesian product of $d-1$ open intervals. The disjoint union

$$
P_{x}:=\bigcup_{y \in Q}(x, y)=\{(1-t) x+t y \mid t \in(0,1), y \in Q\}
$$


forms an open pyramid. Without loss of generality we may assume that $x=0$ and $Q=$ $(-1,1)^{d-1} \times\{1\}$. We define the transformation

$$
g:(-1,1)^{d} \rightarrow P_{x}, \quad\left(s_{1}, s_{2}, \ldots, s_{d}\right) \mapsto \frac{s_{1}+1}{2}\left(s_{2}, s_{3}, \ldots, s_{d}, 1\right) .
$$

The map $g$ is obviously a $C^{1}$-diffeomorphism with inverse

$$
g^{-1}\left(z_{1}, \ldots, z_{d}\right)=\left(2 z_{d}-1, \frac{z_{1}}{z_{d}}, \ldots, \frac{z_{d-1}}{z_{d}}\right) .
$$

Since $P_{x}$ is open in $\mathbb{R}^{d}$ and $C$ is Lebesgue measurable, also $P_{x} \backslash C$ is Lebesgue measurable. In the following we use the notation $s:=\left(s_{1}, \ldots, s_{d}\right)$ and $\tilde{s}:=\left(s_{2}, \ldots, s_{d}\right)$. By the Transformation Theorem and the Theorem of Fubini we obtain

$$
\begin{aligned}
\lambda^{d}\left(P_{x} \backslash C\right) & =\int_{g\left((-1,1)^{d}\right)} \chi_{P_{x} \backslash C}(y) d y \\
& =\int_{(-1,1)^{d}} \chi_{P_{x} \backslash C}(g(s))|\operatorname{det} D g(s)| d s \\
& =\int_{(-1,1)^{d-1}}\left[\int_{0}^{1} \chi_{(x, y) \backslash C}\left(g\left(s_{1}, \tilde{s}\right)\right)\left|\operatorname{det} D g\left(s_{1}, \tilde{s}\right)\right| d s_{1}\right] d \tilde{s} .
\end{aligned}
$$

Now assume to the contrary that the inner integral over $s_{1}$ is zero for some $\tilde{s} \in(-1,1)^{d-1}$. Since $\left|\operatorname{det} D g\left(s_{1}, \tilde{s}\right)\right|>0$ this implies that $\chi_{(x, y) \backslash C}\left(g\left(s_{1}, \tilde{s}\right)\right)=0$ for almost all $s_{1} \in(0,1)$, which is a contradiction to the assumption that the Lebesgue measure of $(x, y) \backslash C$ is positive. Consequently, $\lambda^{d}\left(P_{x} \backslash C\right)>0$, which is a contradiction to (3.2). This shows that $C_{x}$ is dense in $\mathbb{R}^{d}$. Now let $y \in C_{x}$. Define $c(t):=(1-t) x+t y, c:[0,1] \rightarrow \mathbb{R}^{d}$. Since $f$ is Lipschitz continuous by assumption, the curve $f \circ c:[0,1] \rightarrow \mathbb{R}^{d}$ is rectifiable and by $[6$, Theorem 2.7.6., p. 57] the length of $f \circ c$ can be calculated as follows.

$$
\begin{aligned}
\operatorname{length}(f \circ c) & =\int_{0}^{1}\left\|\frac{d}{d t}(f \circ c)(t)\right\| d t=\int_{0}^{1}\left\|D f_{c(t)} \dot{c}(t)\right\| d t \\
& =\int_{0}^{1}\left\|D f_{c(t)}(y-x)\right\| d t .
\end{aligned}
$$

Since $f \circ c$ is a curve from $f(x)$ to $f(y)$ and the estimate (3.1) holds, we obtain

$$
\|f(x)-f(y)\| \leq \operatorname{length}(f \circ c) \leq \int_{0}^{1}\left\|D f_{c(t)}\right\|\|x-y\| d t \leq L\|x-y\| .
$$

From the continuity of the function $y \mapsto \frac{\|f(x)-f(y)\|}{\|x-y\|}, \mathbb{R}^{d} \backslash\{x\} \rightarrow \mathbb{R}$, it follows that the same estimate holds for all $y \in \mathbb{R}^{d}$.

\section{Kinematic similarity}

In this section we study kinematic similarity of linear flows. We use the following definition according to $[\mathbf{8}$, p. 39].

Definition 1. The two linear differential equations

$$
\dot{x}=A(t) x \text { and } \dot{x}=B(t) x
$$

with continuous functions $A, B: \mathbb{R} \rightarrow \mathbb{R}^{d \times d}$ are said to be kinematically similar if there exists a solution $S: \mathbb{R} \rightarrow \mathrm{Gl}(d, \mathbb{R})$ of the differential equation

$$
\dot{S}=B(t) S-S A(t),
$$


such that both $t \mapsto S(t)$ and $t \mapsto S(t)^{-1}$ are bounded. In this case the function $(t, x) \mapsto S(t) x$ is called a kinematic similarity transformation.

REMARK 1. In the autonomous case $\left(A(t) \equiv A\right.$ and $B(t) \equiv B$ for some $\left.A, B \in \mathbb{R}^{d \times d}\right)$ we also say that the matrices $A$ and $B$ and the corresponding flows $e^{A t} x$ and $e^{B t} x$ are kinematically similar.

A straightforward calculation shows that if $\mu(t)$ is a solution of $\dot{x}=A x$ then $\nu(t):=S(t) \mu(t)$ is a solution of $\dot{x}=B x$, provided that $S$ solves (4.1). The following proposition characterizes kinematic similarity in the autonomous case.

Proposition 4.1. Two matrices $A, B \in \mathbb{R}^{d \times d}$ are kinematically similar if and only if there exists $S_{0} \in \mathrm{Gl}(d, \mathbb{R})$ such that both $t \mapsto e^{B t} S_{0} e^{-A t}$ and $t \mapsto e^{A t} S_{0}^{-1} e^{-B t}$ are bounded on $\mathbb{R}$.

Proof. In the autonomous case the solution of the initial value problem $\dot{S}=B S-S A$, $S(0)=S_{0} \in \mathbb{R}^{d \times d}$, is given by $S(t)=e^{B t} S_{0} e^{-A t}$ since

$$
\dot{S}(t)=B e^{B t} S_{0} e^{-A t}-e^{B t} S_{0} e^{-A t} A=B S(t)-S(t) A \text { and } S(0)=S_{0} .
$$

Since $S(t)$ is invertible if and only if $S_{0}$ is invertible, the assertion holds.

REMARK 2. If the matrices $A$ and $B$ are linearly conjugate by a matrix $C \in \operatorname{Gl}(d, \mathbb{R})$, i.e. $C A=B C$, then they are also kinematically similar. In this case a kinematic similarity transformation is given by $(t, x) \mapsto e^{B t} C e^{-A t} x \equiv C x$.

The following proposition gives an algebraic characterization of kinematic similarity. It states that two matrices are kinematically similar if and only if their Jordan forms coincide after deleting the imaginary parts of the eigenvalues.

Proposition 4.2. Two matrices $A, B \in \mathbb{R}^{d \times d}$ are kinematically similar if and only if there exist matrices $D, N, S_{A}, S_{B} \in \mathbb{R}^{d \times d}$ such that the real Jordan forms (as they are described in Section 2) $J_{A}$ and $J_{B}$ of $A$ and $B$, respectively, can be written as

$$
J_{A}=D+S_{A}+N \text { and } J_{B}=D+S_{B}+N,
$$

where $D$ is a diagonal matrix containing the real parts of the eigenvalues, $S_{A}, S_{B}$ are skewsymmetric matrices containing the imaginary parts of the eigenvalues and $N$ is a nilpotent upper triangular matrix.

Proof. $\quad(\Rightarrow)$ : Let $A$ and $B$ be kinematically similar. Since linear conjugacy implies kinematic similarity then also $J_{A}$ and $J_{B}$ are kinematically similar. By [5, Theorem 1, p. 312] the Lyapunov exponents, i.e. the real parts of the eigenvalues, of $J_{A}$ and $J_{B}$ are the same and also their algebraic multiplicities. We denote by $\lambda_{1}<\lambda_{2}<\cdots<\lambda_{r}$ the different real parts and by $m_{i}$ the multiplicity of $\lambda_{i}$ for $i=1, \ldots, r$. We define

$$
D:=\left[\begin{array}{cccc}
\lambda_{1} I_{m_{1}} & & & \\
& \lambda_{2} I_{m_{2}} & & \\
& & \ddots & \\
& & & \lambda_{r} I_{m_{r}}
\end{array}\right],
$$


where $I_{m_{i}}$ is the $m_{i} \times m_{i}$ identity matrix and $m_{1}+\cdots+m_{r}=r$. Then

$$
J_{X}=D+S_{X}+N_{X}, \quad X=A, B
$$

where $S_{X}$ is a skew-symmetric matrix containing the imaginary parts of the eigenvalues of $X$, and $N_{X}$ is the nilpotent part of $J_{X}$. Now let $(t, x) \mapsto S(t) x, S(t)=e^{J_{B} t} S_{0} e^{-J_{A} t}$ be a kinematic similarity transformation from $J_{A}$ to $J_{B}$. Let $x \in \mathbb{R}^{d}$ be a vector contained in the Lyapunov space corresponding to the real part $\lambda_{i}, i \in\{1, \ldots, r\}$. Then $t \mapsto\left\|e^{J_{A} t} x\right\| e^{-\lambda_{i} t}$ has subexponential growth, since

$$
\left\|e^{J_{A} t} x\right\| e^{-\lambda_{i} t}=\left\|e^{S_{A} t} e^{N_{A} t} e^{D t} x\right\| e^{-\lambda_{i} t}=\left\|e^{N_{A} t} e^{\lambda_{i} t} x\right\| e^{-\lambda_{i} t}=\left\|e^{N_{A} t} x\right\| .
$$

Here we used that $e^{S_{A} t}$ is orthogonal for all $t \in \mathbb{R}$. Let $M>0$ be an upper bound for $\|S(t)\|$ and $m>0$ an upper bound for $\left\|S(t)^{-1}\right\|$. Then

$$
\begin{aligned}
m^{-1}\left\|e^{J_{A} t} x\right\| & \leq\left\|S(t)^{-1}\right\|^{-1}\left\|e^{J_{A} t} x\right\| \\
& \leq\left\|S(t) e^{J_{A} t} x\right\| \\
& \leq\|S(t)\|\left\|e^{J_{A} t} x\right\| \leq M\left\|e^{J_{A} t} x\right\| .
\end{aligned}
$$

This shows that also $\left\|S(t) e^{J_{A} t} x\right\|=\left\|e^{J_{B} t} S_{0} x\right\|$ has subexponential growth, which implies that $S(t) x$ is also contained in the Lyapunov space corresponding to $\lambda_{i}$. Thus $S(t)$ must have the same block diagonal form as $D$, and consequently $S_{0} D=D S_{0}$. This yields

$$
\left\|e^{J_{B} t} S_{0} e^{-J_{A} t}\right\|=\left\|e^{S_{B} t} e^{N_{B} t} e^{D t} S_{0} e^{-D t} e^{-N_{A} t} e^{-S_{A} t}\right\|=\left\|e^{N_{B} t} S_{0} e^{-N_{A} t}\right\|,
$$

since $e^{S_{A} t}$ and $e^{S_{B} t}$ are orthogonal for all $t \in \mathbb{R}$. Hence $N_{A}$ and $N_{B}$ are kinematically similar. Obviously, every entry of the matrix $\tilde{S}(t):=e^{N_{B} t} S_{0} e^{-N_{A} t}$ is a polynomial in $t$. Since $\|\tilde{S}(t)\|$ is bounded, this implies that every entry is constant, i.e. $\tilde{S}(t) \equiv S_{0}$ and consequently

$$
e^{N_{B} t} S_{0}=S_{0} e^{N_{A} t}
$$

By differentiating this equation at $t=0$ we obtain $S_{0} N_{A}=N_{B} S_{0}$. Thus we may assume $N_{A}=N_{B}$, which yields the desired result.

$(\Leftarrow)$ : It suffices to show that $J_{A}=D+S_{A}+N$ and $J_{B}=D+S_{B}+N$ are kinematically similar. Since skew-symmetric $2 \times 2$-matrices commute, $S_{A}$ and $S_{B}$ commute, and consequently also $J_{A}$ and $J_{B}$. We define

$$
S(t):=e^{J_{B} t} I e^{-J_{A} t}=e^{\left(J_{B}-J_{A}\right) t}=e^{\left(S_{B}-S_{A}\right) t} .
$$

Then $\|S(t)\|=\left\|S_{0}\right\|$ and $\left\|S(t)^{-1}\right\|=\left\|S_{0}^{-1}\right\|$ for all $t \in \mathbb{R}$, since $S(t)$ is orthogonal. This proves the kinematic similarity of $J_{A}$ and $J_{B}$.

The proof of Proposition 4.2 yields the following corollary.

Corollary 4.3. If $(t, x) \mapsto S(t) x$ with $S(t)=e^{B t} S_{0} e^{-A t}$ is a kinematic similarity transformation for the two Jordan matrices $J_{A}=D+S_{A}+N, J_{B}=D+S_{B}+N$ in (4.2), then

$$
S_{0} D=D S_{0} \text { and } S_{0} N=N S_{0} .
$$

\section{The main result}

Now we introduce the notion of Lipschitz conjugacy for linear flows. 
Definition 2. The linear flows $e^{A t} x$ and $e^{B t} x$ are said to be Lipschitz conjugate if there exists a bi-Lipschitz homeomorphism $h: \mathbb{R}^{d} \rightarrow \mathbb{R}^{d}$ which is a topological conjugacy from $e^{A t} x$ to $e^{B t} x$. The map $h$ is then called a Lipschitz conjugacy.

Lemma 5.1. Let $h: \mathbb{R}^{d} \rightarrow \mathbb{R}^{d}$ be a topological conjugacy from $e^{A t} x$ to $e^{B t} x$. Then the set $\Delta(h)$ is the union of orbits of the flow $e^{A t} x$.

Proof. We have to show that $h$ is differentiable at a point $x \in \mathbb{R}^{d}$ if and only if $h$ is differentiable at all points of the orbit $O_{x}=\left\{e^{A t} x\right\}_{t \in \mathbb{R}}$. This follows easily from the conjugacy identity, which can be written as $h=e^{-B t} \circ h \circ e^{A t}$. Applying the chain rule to this equation yields

$$
D h_{e^{-A t} x}=e^{-B t} D h_{x} e^{A t} .
$$

Thus differentiability at $x$ implies differentiability at $e^{-A t} x$ for every $t \in \mathbb{R}$.

Lemma 5.2. Let $F \in \mathbb{R}^{d \times d}$. Then there exists a subspace $V$ of $\mathbb{R}^{d}$ and a norm $\|\cdot\|_{*}$ on $\mathbb{R}^{d}$ such that the following statements hold.

(i) $\left\|e^{F t} x\right\|_{*}=\|x\|_{*}$ for all $x \in V$ and $t \in \mathbb{R}$.

(ii) If a vector $x \in \mathbb{R}^{d}$ satisfies

$$
m \leq\left\|e^{F t} x\right\| \leq M \text { for all } t \geq 0
$$

with constants $m, M \geq 0$, then $x \in V$.

Proof. For any eigenvalue $\lambda$ of $F$ let $E_{\lambda}^{\mathbb{C}}(F)$ denote the corresponding complex eigenspace,

$$
E_{\lambda}^{\mathbb{C}}(F)=\left\{v \in \mathbb{C}^{d} \mid F v=\lambda v\right\}
$$

Let $E_{i \alpha}(F):=\left(E_{i \alpha}^{\mathbb{C}} \oplus E_{-i \alpha}^{\mathbb{C}}\right) \cap \mathbb{R}^{d}$ for every pair $\pm i \alpha$ of complex conjugate imaginary eigenvalues and let $E_{0}(F)=E_{0}^{\mathbb{C}}(F) \cap \mathbb{R}^{d}$. We define

$$
V:=\bigoplus_{\operatorname{Re}(\lambda)=0} E_{\lambda}(F)=E_{\lambda_{1}}(F) \oplus \cdots \oplus E_{\lambda_{r}}(F) .
$$

Let $C \in \operatorname{Gl}(d, \mathbb{R})$ be a matrix such that $J_{F}:=C F C^{-1}$ is the real Jordan form of $F$. Then $C$ maps eigenvectors of $F$ to eigenvectors of $J_{F}$, and eigenvectors of $J_{F}$ corresponding to different eigenvalues are orthogonal with respect to the Euclidean scalar product. We define the norm $\|\cdot\|_{*}$ by $\|x\|_{*}:=\|C x\|$ for all $x \in \mathbb{R}^{d}$. Now we can show that $V$ has the desired properties:

(i) Let $x \in V$. Then there exist unique $x_{i} \in E_{\lambda_{i}}, i=1, \ldots, r$, such that $x=x_{1}+\cdots+x_{r}$. The restriction of $e^{J_{F} t}$ to each eigenspace $E_{\lambda}(F)$ with $\operatorname{Re}(\lambda)=0$ is an isometry with respect to the Euclidean norm. This yields

$$
\begin{aligned}
\left\|e^{F t} x\right\|_{*}^{2} & =\left\|C e^{F t} x\right\|^{2}=\left\|e^{J t} C x\right\|^{2}=\left\|e^{J t}\left(C x_{1}+\cdots+C x_{r}\right)\right\|^{2} \\
& =\left\|e^{J t} C x_{1}\right\|^{2}+\cdots+\left\|e^{J t} C x_{r}\right\|^{2}=\left\|C x_{1}\right\|^{2}+\cdots+\left\|C x_{r}\right\|^{2} \\
& =\left\|C\left(x_{1}+\cdots+x_{r}\right)\right\|^{2}=\|C x\|^{2}=\|x\|_{*}^{2}
\end{aligned}
$$

for every $t \in \mathbb{R}$.

(ii) Let $x \in \mathbb{R}^{d}$ be a vector which satisfies (5.1). Then $\left\|e^{F t} x\right\|$ does neither converge to zero nor to infinity for $t \rightarrow \infty$. Thus $x \in \mathbb{E}^{c}(F)$. Also $\left\|e^{F t} x\right\|$ does not grow polynomially, and hence $x$ must be a linear combination of eigenvectors corresponding to eigenvalues with real part zero. 
The following proposition and its corollary show that in the hyperbolic case Lipschitz conjugacy is equivalent to conjugacy by a homeomorphism which, together with its inverse, satisfies a global Lipschitz condition.

Proposition 5.3. Let $A, B \in \mathbb{R}^{d \times d}$ be matrices with negative eigenvalue real parts. Let $h: \mathbb{R}^{d} \rightarrow \mathbb{R}^{d}$ be a topological conjugacy from $e^{A t} x$ to $e^{B t} x$. Then the following statements are equivalent:

(i) $h$ is a bi-Lipschitz homeomorphism.

(ii) $h$ and $h^{-1}$ satisfy global Lipschitz conditions, i.e. there exist constants $l, L>0$ with

$$
l\|x-y\| \leq\|h(x)-h(y)\| \leq L\|x-y\| \text { for all } x, y \in \mathbb{R}^{d} .
$$

(iii) $h$ and $h^{-1}$ satisfy a Lipschitz condition in a neighborhood $U$ of $x=0$.

Proof. Obviously the implications "(ii) $\Rightarrow$ (i)" and "(i) $\Rightarrow$ (iii)" hold. Hence we only have to show that (iii) implies (ii): Let $U$ be an open neighborhood of $x=0$ such that $\left.h\right|_{U}$ and $\left.h^{-1}\right|_{U}$ are Lipschitz continuous with Lipschitz constants $L_{ \pm}>0$. By choosing $U$ small enough we obtain that

$$
L_{-}^{-1}\|x-y\| \leq\|h(x)-h(y)\| \leq L_{+}\|x-y\| \text { for all } x, y \in U .
$$

We want to show that $h$ is Lipschitz continuous. To this end, let $x_{0} \in \mathbb{R}^{d} \backslash\{0\}$. Since all eigenvalue real parts of $A$ are negative by assumption, the flow $e^{A t} x$ is contracting, i.e. every trajectory converges to zero in forward time. Therefore there exists a time $t_{0} \geq 0$ with $e^{A t_{0}} x_{0} \in U$. Then $V:=e^{-A t_{0}} U$ is an open neighborhood of $x$ and for any $z_{1}, z_{2} \in V$ there exist $x_{1}, x_{2} \in U$ with $z_{1}=e^{-A t_{0}} x_{1}$ and $z_{2}=e^{-A t_{0}} x_{2}$. Consequently,

$$
\begin{aligned}
\left\|h\left(z_{1}\right)-h\left(z_{2}\right)\right\| & =\left\|h\left(e^{-A t_{0}} x_{1}\right)-h\left(e^{-A t_{0}} x_{2}\right)\right\| \\
& =\left\|e^{-B t_{0}} h\left(x_{1}\right)-e^{-B t_{0}} h\left(x_{2}\right)\right\| \\
& =\left\|e^{-B t_{0}}\left(h\left(e^{A t_{0}} z_{1}\right)-h\left(e^{A t_{0}} z_{2}\right)\right)\right\| \\
& \leq L_{+}\left\|e^{-B t_{0}}\right\|\left\|e^{A t_{0}}\right\|\left\|z_{1}-z_{2}\right\| .
\end{aligned}
$$

This proves Lipschitz continuity of $h$. In order to apply Lemma 3.2 and to obtain the result we have to prove that $D h_{x}$ is globally bounded. To this end, let $\xi \in \Delta\left(\left.h\right|_{U}\right)$. Then there exists a function $r: \mathbb{R}^{d} \rightarrow \mathbb{R}^{d}$ with $\lim _{x \rightarrow \xi} \frac{r(x)}{\|x-\xi\|}=0$ and for every $x \in \mathbb{R}^{d} \backslash\{\xi\}$ we have

$$
\frac{\|h(x)-h(\xi)\|}{\|x-\xi\|}=\left\|D h_{\xi} \frac{x-\xi}{\|x-\xi\|}+\frac{r(x)}{\|x-\xi\|}\right\| \text {. }
$$

This implies

$$
L_{-}^{-1} \leq\left\|D h_{\xi} v\right\| \leq L_{+} \text {for all } v \in \mathbb{R}^{d} \text { with }\|v\|=1,
$$

and hence $L_{-}^{-1} \leq\left\|D h_{\xi}\right\| \leq L_{+}$. Since the flow $e^{A t} x$ is contracting, we may assume that $U$ is forward invariant under $e^{\bar{A} t} x$, i.e. $e^{A t} U \subset U$ for all $t \geq 0$. Consequently,

$$
\left\|D h_{e^{A t} \xi}\right\|=\left\|e^{B t} D h_{\xi} e^{-A t}\right\| \in\left[L_{-}^{-1}, L_{+}\right] \text {for all } t \geq 0 .
$$

Since $t \mapsto e^{B t} D h_{\xi} e^{-A t}$ is the trajectory through $D h_{\xi}$ of the linear flow induced by the matrix differential equation $\dot{S}=B S-S A$, we obtain by Lemma 5.2 a norm $\|\cdot\|_{*}$ on $\mathbb{R}^{d \times d}$ such that

$$
\left\|e^{B t} D h_{\xi} e^{-A t}\right\|_{*}=\left\|D h_{\xi}\right\|_{*} \text { for all } t \in \mathbb{R} \text { and } \xi \in \Delta\left(\left.h\right|_{U}\right) \text {. }
$$

Since all norms on $\mathbb{R}^{d \times d}$ are equivalent, there are $c, C>0$ such that

$$
c\|X\| \leq\|X\|_{*} \leq C\|X\| \text { for all } X \in \mathbb{R}^{d \times d} .
$$


This implies that for $x=e^{A t} \xi$ with $\xi \in \Delta\left(\left.h\right|_{U}\right)$ we have

$$
\left\|D h_{x}\right\| \leq \frac{1}{c}\left\|D h_{x}\right\|_{*}=\frac{1}{c}\left\|D h_{e^{A t} \xi}\right\|_{*}=\frac{1}{c}\left\|D h_{\xi}\right\|_{*} \leq \frac{C}{c}\left\|D h_{\xi}\right\| \leq \frac{C}{c} L_{+} .
$$

Consequently, Lemma 3.2 can be applied and guarantees that $h$ satisfies a global Lipschitz condition. The same arguments can be applied to show that $h^{-1}$ satisfies a global Lipschitz condition.

Corollary 5.4. Let $A, B \in \mathbb{R}^{d \times d}$ be matrices with eigenvalues real parts different from zero. Then the following statements are equivalent:

(i) $e^{A t} x$ and $e^{B t} x$ are Lipschitz conjugate.

(ii) There exists a topological conjugacy from $e^{A t} x$ to $e^{B t} x$ which, together with its inverse, satisfies a global Lipschitz condition.

Proof. Assume that (i) holds with a Lipschitz conjugacy $h: \mathbb{R}^{d} \rightarrow \mathbb{R}^{d}$. By the dynamical characterization of stable and unstable eigenspaces it is clear that $h\left(\mathbb{E}^{\sigma}(A)\right)=\mathbb{E}^{\sigma}(B)$ for $\sigma=$ $s, u$. By Theorem 5.3 the restrictions $h^{\sigma}:=\left.h\right|_{\mathbb{E}^{\sigma}(A)}, \sigma=s, u$, satisfy global Lipschitz conditions. Define

$$
\tilde{h}(x):=h^{s}\left(P^{s}(x)\right)+h^{u}\left(P^{u}(x)\right), \quad \tilde{h}: \mathbb{R}^{d} \rightarrow \mathbb{R}^{d},
$$

where $P^{s}$ and $P^{u}$ are the projections onto $\mathbb{E}^{s}(A)$ and $\mathbb{E}^{u}(A)$, respectively. It is easy to see that $\tilde{h}$ is a homeomorphism, and

$$
\tilde{h}\left(e^{A t} x\right)=h^{s}\left(e^{A t} x^{s}\right)+h^{u}\left(e^{A t} x^{u}\right)=e^{B t} h^{s}\left(x^{s}\right)+e^{B t} h^{u}\left(x^{u}\right)=e^{B t} \tilde{h}(x) .
$$

Hence also $\tilde{h}$ is a topological conjugacy from $e^{A t} x$ to $e^{B t} x$. The global Lipschitz continuity of $h^{s}$ and $h^{u}$ yield the global Lipschitz continuity of $\tilde{h}$. To see this, let $L_{s}$ be a Lipschitz constant for $h^{s}$ and $L_{u}$ one for $h^{u}$. Then for all $x, y \in \mathbb{R}^{d}$

$$
\begin{aligned}
\|\tilde{h}(x)-\tilde{h}(y)\| & \leq\left\|\tilde{h}\left(P^{s}(x)\right)-\tilde{h}\left(P^{s}(y)\right)\right\|+\left\|\tilde{h}\left(P^{u}(x)\right)-\tilde{h}\left(P^{u}(y)\right)\right\| \\
& \leq L_{s}\left\|P^{s}\right\|\|x-y\|+L_{u}\left\|P^{u}\right\|\|x-y\| \\
& =\left(L_{s}\left\|P^{s}\right\|+L_{u}\left\|P^{u}\right\|\right)\|x-y\| .
\end{aligned}
$$

This proves the claim.

By Corollary 5.4 we may assume in the following that all Lipschitz conjugacies satisfy global Lipschitz conditions. In order to prove our main theorem we need the following technical lemma.

LEMmA 5.5. Let $A, B \in \mathbb{R}^{d \times d}$ be matrices with only negative (or only positive) eigenvalue real parts. Let $h: \mathbb{R}^{d} \rightarrow \mathbb{R}^{d}$ be a bi-Lipschitz homeomorphism which satisfies

$$
B h(x)=D h_{x} A x \text { for all } x \in \Delta(h) .
$$

Then $h$ is a Lipschitz conjugacy from $e^{A t} x$ to $e^{B t} x$.

Proof. If the eigenvalues of $A$ and $B$ have positive real parts, we can replace $A$ by $-A$ and $B$ by $-B$. Consequently, we may assume that the eigenvalue real parts are negative. We define the function

$$
f: \mathbb{R} \times \mathbb{R}^{d} \rightarrow \mathbb{R}^{d}, \quad(t, x) \mapsto h\left(e^{A t} x\right)-e^{B t} h(x) .
$$

Since $f$ is obviously continuous, the set

$$
X:=f^{-1}(0)=\left\{(t, x) \in \mathbb{R} \times \mathbb{R}^{d} \mid h\left(e^{A t} x\right)=e^{B t} h(x)\right\}
$$


is closed. Consequently, it suffices to show that $X$ is dense in $\mathbb{R} \times \mathbb{R}^{d}$. By $[\mathbf{1}$, Theorem 5.1., p. 108] there exists a so-called adapted norm $\|\cdot\|_{A}$, i.e. a norm with the property

$$
\left\|e^{A t} x\right\|_{A} \leq e^{-a t}\|x\|_{A} \text { for all } x \in \mathbb{R}^{d} \text { and } t \geq 0,
$$

where $a>0$ is a constant. According to [1] it is possible to define such a norm by

$$
\|x\|_{A}:=\int_{0}^{\tau} e^{a s}\left\|e^{A s} x\right\| d s \text { for all } x \in \mathbb{R}^{d},
$$

where $a, \tau>0$ are suitably chosen constants. We define the unit sphere $S_{A}:=\left\{x \in \mathbb{R}^{d} \mid\|x\|_{A}=\right.$ $1\}$ in the norm $\|\cdot\|_{A} \cdot S_{A}$ is a fundamental domain for the restriction of the flow $e^{A t} x$ to $\mathbb{R}^{d} \backslash\{0\}$, which means that for every $x \neq 0$ the orbit $O_{x}=\left\{e^{A t} x\right\}_{t \in \mathbb{R}}$ hits $S_{A}$ exactly once. $S_{A}$ is also a differentiable $(d-1)$-dimensional submanifold of $\mathbb{R}^{d}$. In order to show this, let $F(x):=\|x\|_{A}-1, F: \mathbb{R}^{d} \rightarrow \mathbb{R}$. Since

$$
D F_{x}=x^{T} \int_{0}^{\tau} e^{a s} \frac{e^{A^{T} s} e^{A s}}{\left\|e^{A s} x\right\|} d s=: x^{T} \tilde{A}(x) \text { for all } x \in \mathbb{R}^{d} \backslash\{0\},
$$

and $\tilde{A}(x)$ is a positive definite symmetric matrix, $S_{A}$ is a regular preimage. Now assume to the contrary that there exists an open set $U \subset S_{A}$ such that for all $z \in U$

$$
\lambda^{1}\left(\left\{t \in \mathbb{R} \mid e^{A t} z \notin \Delta(h)\right\}\right)=\int_{-\infty}^{\infty} \chi_{O_{z} \backslash \Delta(h)}\left(e^{A t} z\right) d t>0 .
$$

If this set is not Lebesgue measurable for every $z \in U$, we can replace $\Delta(h)$ by an $F_{\sigma}$-set of the same measure as in the proof of Lemma 3.2. We define the transformation

$$
g: \mathbb{R} \times S_{A} \rightarrow \mathbb{R}^{d} \backslash\{0\}, \quad g(t, z):=e^{A t} z .
$$

The map $g$ is a $C^{1}$-diffeomorphism with inverse

$$
g^{-1}(x)=\left(-\tau(x), e^{A \tau(x)} x\right),
$$

where $\tau: \mathbb{R}^{d} \backslash\{0\} \rightarrow \mathbb{R}$ is defined implicitely by $\left\|e^{A \tau(x)} x\right\|_{A}-1=0$. The following holds for the measure of $\mathbb{R}^{d} \backslash \Delta(h)$.

$$
\begin{aligned}
& \lambda^{d}\left(\mathbb{R}^{d} \backslash \Delta(h)\right)=\int_{\mathbb{R}^{d}} \chi_{\mathbb{R}^{d} \backslash \Delta(h)}(x) d x=\int_{\mathbb{R}^{d} \backslash\{0\}} \chi_{\mathbb{R}^{d} \backslash \Delta(h)}(x) d x=\int_{g\left(\mathbb{R} \times S_{A}\right)} \chi_{\mathbb{R}^{d} \backslash \Delta(h)}(x) d x \\
= & \int_{\mathbb{R} \times S_{A}} \chi_{\mathbb{R}^{d} \backslash \Delta(h)}(g(t, z))|\operatorname{det} D g(t, z)| d(t, z)=\int_{S_{A}}\left[\int_{\mathbb{R}} \chi_{\mathbb{R}^{d} \backslash \Delta(h)}\left(e^{A t} z\right)|\operatorname{det} D g(t, z)| d t\right] d z \\
\geq & \int_{U}\left[\int_{-\infty}^{\infty} \chi_{\mathbb{R}^{d} \backslash \Delta(h)}\left(e^{A t} z\right)|\operatorname{det} D g(t, z)| d t\right] d z .
\end{aligned}
$$

Assume to the contrary that the inner integral is zero for one $z \in U$. Then $\chi_{\mathbb{R}^{d} \backslash \Delta(h)}\left(e^{A t} z\right)=$ 0 for almost all $t \in \mathbb{R}$ since $|\operatorname{det} D g(t, z)|>0$, which yields a contradiction to (5.6). Hence $\lambda^{d}\left(\mathbb{R}^{d} \backslash \Delta(h)\right)>0$, which is a contradiction to Theorem 3.1. Thus there exists a dense set $Z \subset S_{A}$ such that for every $z \in Z$ the derivative $D h_{e^{A t} z}$ exists for almost every $t \in \mathbb{R}$. Let $z \in Z$. We define the curves $\gamma_{z}(t):=e^{A t} z$ and $\tilde{\gamma}_{z}:=h \circ \gamma_{z}$. Then equation (5.4) yields

$$
B \tilde{\gamma}_{z}(t)=B h\left(\gamma_{z}(t)\right)=D h_{\gamma_{z}(t)} \dot{\gamma}_{z}(t)=\dot{\tilde{\gamma}}_{z}(t) \text { for almost all } t \in \mathbb{R}
$$

Since $h$ is Lipschitz continuous, also $\tilde{\gamma}_{z}$ is Lipschitz continuous and therefore absolutely continuous. This implies that $\dot{\tilde{\gamma}}_{z}$ is Lebesgue integrable on every compact interval $[0, t]$ and

Consequently,

$$
\tilde{\gamma}_{z}(t)-\tilde{\gamma}_{z}(0)=\int_{0}^{t} \dot{\tilde{\gamma}}_{z}\left(t^{\prime}\right) d t^{\prime} \text { for all } t \in \mathbb{R} .
$$

$$
\tilde{\gamma}_{z}(t)=\tilde{\gamma}_{z}(0)+\int_{0}^{t} \tilde{B}_{\tilde{\gamma}}\left(t^{\prime}\right) d t^{\prime} \text { for all } t \in \mathbb{R}
$$


and therefore $\tilde{\gamma}_{z}$ is continuously differentiable. This implies that the linear differential equation (5.7) is satisfied for all $t \in \mathbb{R}$, and thus

$$
h\left(e^{A t} z\right)=\tilde{\gamma}_{z}(t)=e^{B t} h\left(\gamma_{z}(0)\right)=e^{B t} h(z) \text { for all } t \in \mathbb{R} .
$$

If $x=e^{A s} z$ for some $s \in \mathbb{R}$ and $z \in Z$ we get

$$
\begin{aligned}
h\left(e^{A t} z\right) & =h\left(e^{A(t+s)} z\right)=e^{B(t+s)} h(z) \\
& =e^{B t} e^{B s} h(z)=e^{B t} h\left(e^{A s} z\right)=e^{B t} h(x) .
\end{aligned}
$$

This shows that $X$ contains the set $\mathbb{R} \times \bigcup_{z \in Z} O_{z}$, which is obviously dense in $\mathbb{R} \times \mathbb{R}^{d}$. Thus $X$ is dense in $\mathbb{R} \times \mathbb{R}^{d}$, which proves the lemma.

Now we can state and prove our main theorem.

TheOREM 5.6. Let $A, B \in \mathbb{R}^{d \times d}$ be matrices with eigenvalues real parts different from zero. Then the following statements are equivalent:

(i) $e^{A t} x$ and $e^{B t} x$ are Lipschitz conjugate.

(ii) The real Jordan forms $J_{A}$ and $J_{B}$ of $A$ and $B$, respectively, can be written as

$$
J_{A}=D+S_{A}+N, \quad J_{B}=D+S_{B}+N,
$$

where $D$ is a diagonal matrix containing the real parts of the eigenvalues, $S_{A}, S_{B}$ are skew-symmetric matrices containing the imaginary parts of the eigenvalues and $N$ is a nilpotent upper triangular matrix, and, furthermore, the following relation holds:

$$
N\left(S_{B}-S_{A}\right)=0 .
$$

Proof. (i) $\Rightarrow$ (ii): Let $h: \mathbb{R}^{d} \rightarrow \mathbb{R}^{d}$ be a Lipschitz conjugacy from $e^{A t} x$ to $e^{B t} x$. By Corollary 5.4 we may assume that $h$ and $h^{-1}$ satisfy global Lipschitz conditions with corresponding Lipschitz constants $L_{ \pm}>0$. Let $\xi \in \Delta(h)$. Then by the proof of Proposition 5.3 (Formula (5.3)) we get

$$
L_{-}^{-1} \leq\left\|D h_{\xi} v\right\| \leq L_{+} \text {for all } v \in \mathbb{R}^{d} \text { with }\|v\|=1,
$$

which implies both

$$
\left\|e^{B t} D h_{\xi} e^{-A t}\right\|=\left\|D h_{e^{A t} \xi}\right\| \leq L_{+}
$$

and

$$
\left\|e^{A t} D h_{\xi}^{-1} e^{-B t}\right\|=\left\|\left(D h_{e^{A t} \xi}\right)^{-1}\right\| \leq L_{-}
$$

for all $t \in \mathbb{R}$. Thus, by Proposition 4.1, $A$ and $B$ are kinematically similar. We may assume that $A$ and $B$ are given in real Jordan form, and therefore by Proposition 4.2 we obtain

$$
A=D+S_{A}+N \text { and } B=D+S_{B}+N
$$

where $D$ is a diagonal matrix containing the real parts of the eigenvalues, $S_{A}, S_{B}$ are skewsymmetric matrices containing the imaginary parts of the eigenvalues and $N$ is a nilpotent upper triangular matrix. Without loss of generality we may assume that $D=\lambda I$ for some $\lambda \in \mathbb{R} \backslash\{0\}$, since $h$ surely preserves the Lyapunov spaces. Differentiating the conjugacy identity $h\left(e^{A t} x\right)=e^{B t} h(x)$ by $t$ at $t=0$ yields

$$
B h(x)=D h_{x} A x \text { for all } x \in \Delta(h) .
$$

The matrix $C:=\lambda I+N$ is invertible and multiplication by $C^{-1}$ from the left transforms $(5.9)$ into

$$
\left(I+C^{-1} S_{B}\right) h(x)=C^{-1} D h_{x}\left(C+S_{A}\right) x \text { for all } x \in \Delta(h)
$$


By Corollary 4.3 we obtain $C D h_{x}=D h_{x} C$ and hence $D h_{x} C^{-1}=C^{-1} D h_{x}$. It follows that equation (5.10) is equivalent to

$$
\left(I+C^{-1} S_{B}\right) h(x)=D h_{x}\left(I+C^{-1} S_{A}\right) x \text { for all } x \in \Delta(h) .
$$

Since all eigenvalues of $I+C^{-1} S_{X}(X=A, B)$ have real part 1, by Lemma $5.5 h$ is also a Lipschitz conjugacy from $e^{\left(I+C^{-1} S_{A}\right) t} x$ to $e^{\left(I+C^{-1} S_{B}\right) t} x$, which implies the kinematic similarity of $I+C^{-1} S_{A}$ and $I+C^{-1} S_{B}$. Since $e^{\left(I+C^{-1} S_{X}\right) t}=e^{t} e^{C^{-1} S_{X} t}(X=A, B)$ we obtain

$$
e^{\left(I+C^{-1} S_{B}\right) t} D h_{x} e^{-\left(I+C^{-1} S_{A}\right) t}=e^{C^{-1} S_{B} t} D h_{x} e^{-C^{-1} S_{A} t},
$$

and thus, $C^{-1} S_{A}$ and $C^{-1} S_{B}$ are kinematically similar. $C^{-1}$ can be calculated by using the geometric series formula:

$$
\begin{aligned}
C^{-1} & =(\lambda I+N)^{-1}=\lambda^{-1}\left(I+\lambda^{-1} N\right)^{-1} \\
& =\lambda^{-1} \sum_{k=0}^{\infty}\left(-\lambda^{-1} N\right)^{k}=\lambda^{-1} \sum_{k=0}^{d-1}\left(-\lambda^{-1} N\right)^{k} .
\end{aligned}
$$

The latter equality holds since $N^{k}=0$ for all $k \geq d$. This yields

$$
C^{-1} S_{X}=\lambda^{-1} S_{X}+\sum_{k=1}^{d-1} \lambda^{-(k+1)}(-N)^{k} S_{X} \quad(X=A, B) .
$$

The nilpotent part in the Jordan partition of $I+C^{-1} S_{X}$ is therefore

$$
\tilde{M}_{X}:=\tilde{N} S_{X} \text { with } \tilde{N}:=\sum_{k=1}^{d-1} \lambda^{-1}\left(-\lambda^{-1} N\right)^{k}
$$

which follows from the fact that $S_{X}$ and $N$ commute. Consequently $\tilde{M}_{A}$ and $\tilde{M}_{B}$ are kinematically similar, and a kinematic similarity transformation is given by $(t, x) \mapsto S(t) x$ with $S(t)=e^{\tilde{M}_{B} t} D h_{x} e^{-\tilde{M}_{A} t}$. Since $\tilde{M}_{A}$ and $\tilde{M}_{B}$ are nilpotent, every entry of $S(t)$ is a polynomial in $t$, which implies $S(t) \equiv D h_{x}$. Consequently

$$
D h_{x} e^{\tilde{M}_{A} t}=e^{\tilde{M}_{B} t} D h_{x} \Rightarrow D h_{x} \tilde{M}_{A}=\tilde{M}_{B} D h_{x} .
$$

Since $D h_{x}$ and $N$ commute, we also get $D h_{x} \tilde{N}=\tilde{N} D h_{x}$ and thus

$$
\begin{aligned}
0 & =D h_{x} \tilde{M}_{A}-\tilde{M}_{B} D h_{x}=D h_{x} \tilde{N} S_{A}-\tilde{N} S_{B} D h_{x} \\
& =\tilde{N}\left(D h_{x} S_{A}-S_{B} D h_{x}\right) .
\end{aligned}
$$

This means that the image of $D h_{x} S_{A}-S_{B} D h_{x}$ is contained in the kernel of $\tilde{N}$. We want to show that $\operatorname{ker} \tilde{N} \subset \operatorname{ker} N$ and therefore

$$
N\left(D h_{x} S_{A}-S_{B} D h_{x}\right)=\left(D h_{x} S_{A}-S_{B} D h_{x}\right) N=0 .
$$

To this end, let $\tilde{N} x=0$. Since $\tilde{N}=C^{-1}-\lambda^{-1} I$, we obtain

$$
\begin{aligned}
\left(C^{-1}-\lambda^{-1} I\right) x=0 & \Rightarrow x=\lambda^{-1} C x \\
& \Rightarrow x=\left(I+\lambda^{-1} N\right) x \Rightarrow N x=0 .
\end{aligned}
$$

Equation (5.12) says that $S_{A}$ and $S_{B}$ are conjugate on the image of $N$. In particular, the following diagram commutes, since the matrices $S_{A}, S_{B}$ and $D h_{x}$ commute with $N$ :

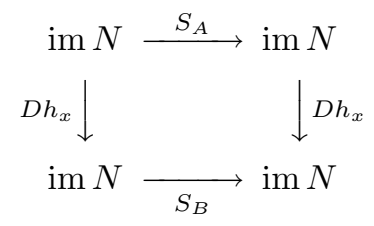


We can write the matrices $S_{A}$ and $S_{B}$ in the following way:

$$
\left.S_{X}=\operatorname{diag}\left(S_{1}(X), \ldots, S_{r}(X)\right)\right), \quad X=A, B,
$$

where $r$ is the number of Jordan blocks in $A$ and $B$ and

$$
S_{k}(X)=\left[\begin{array}{ccccc}
0 & \mu_{k}(X) & & & \\
-\mu_{k}(X) & 0 & & & \\
& & \ddots & & \\
& & & 0 & \mu_{k}(X) \\
& & & -\mu_{k}(X) & 0
\end{array}\right] \in \mathbb{R}^{2 r_{k} \times 2 r_{k}} .
$$

for $k=1, \ldots, s$ and $S_{k}(X)=[0]_{r_{k} \times r_{k}}$ (the $r_{k} \times r_{k}$ zero matrix) for $k=s+1, \ldots, r$. For the numbers $r_{k}$ we have

$$
\sum_{k=1}^{s} 2 r_{k}+\sum_{k=s+1}^{r} r_{k}=d
$$

The nilpotent matrix $N$ is then given by $N=\operatorname{diag}\left(N_{1}, \ldots, N_{r}\right)$ with

$$
N_{k}=\left[\begin{array}{ccccccc}
0 & & 1 & & & & \\
& 0 & & 1 & & & \\
& & \cdot & & \cdot & & \\
& & & \cdot & & 1 & \\
& & & & \cdot & & 1 \\
& & & & & 0 & \\
& & & & & & 0
\end{array}\right] \in \mathbb{R}^{2 r_{k} \times 2 r_{k}} \text { for } k=1, \ldots, s
$$

and

$$
N_{k}=\left[\begin{array}{ccccc}
0 & 1 & & & \\
& \cdot & \cdot & & \\
& & \cdot & \cdot & \\
& & & \cdot & 1 \\
& & & & 0
\end{array}\right] \in \mathbb{R}^{r_{k} \times r_{k}} \text { for } k=s+1, \ldots, r .
$$

With every $k \in\{1, \ldots, r\}$ a subspace $\mathcal{L}_{k} \subset \mathbb{R}^{d}$ is associated such that $\left.S_{X}\right|_{\mathcal{L}_{k}}=S_{k}(X)$ and $\left.N\right|_{\mathcal{L}_{k}}=N_{k} . \mathcal{L}_{k}$ contains the subspace $\mathcal{N}_{k}:=\operatorname{im} N_{k}$ and it holds that

$$
\operatorname{im} N=\mathcal{N}_{1} \oplus \mathcal{N}_{2} \oplus \cdots \oplus \mathcal{N}_{r} .
$$

Obviously, $\operatorname{dim} \mathcal{N}_{k}=\operatorname{dim} \mathcal{L}_{k}-2$ for $k=1, \ldots, s$ and $\operatorname{dim} \mathcal{N}_{k}=\operatorname{dim} \mathcal{L}_{k}-1$ for $k=s+1, \ldots, r$. Consequently, the subspace $\mathcal{N}_{k}$ is trivial if and only if $\operatorname{dim} \mathcal{L}_{k}=2$ and $1 \leq k \leq s$ or $\operatorname{dim} \mathcal{L}_{k}=1$ and $s<k \leq r$. Thus the restriction of $S_{X}$ to $\operatorname{im} N$ has the same eigenvalues as $S_{X}$ except for those whose associated Jordan blocks are all trivial (which means they have size $2 \times 2$ in the nonreal and $1 \times 1$ in the real case). Since $S_{A}$ and $S_{B}$ are linearly conjugate on $\operatorname{im} N$ we may assume that $\mu_{k}(A)=\mu_{k}(B)$ for all $k \in\{1, \ldots, r\}$ with $\mathcal{N}_{k} \neq\{0\}$. This implies $\operatorname{ker}\left(S_{B}-S_{A}\right) \subset \operatorname{im} N$ and thus (5.8) holds.

(ii) $\Rightarrow$ (i): It suffices to show that $e^{J_{A} t} x$ and $e^{J_{B} t} x$ are Lipschitz conjugate, so we may assume $A=J_{A}$ and $B=J_{B}$. We define the Lipschitz conjugacy Lyapunov blockwise. This means that we may also assume that $D=\lambda I$ for one eigenvalue real part $\lambda \neq 0$. Define $S:=S_{B}-S_{A}$. Since $S$ is skew-symmetric, $\mathbb{R}^{d}$ can be written as the direct sum of the kernel and the image of $S$ :

$$
\mathbb{R}^{d}=\operatorname{ker} S \oplus \operatorname{im} S .
$$

Let $U:=\operatorname{ker} S$ and $V:=\operatorname{im} S . U$ and $V$ are invariant with respect to both of the flows $e^{A t} x$ and $e^{B t} x$, since $S$ commutes with $A$ and $B$ by the assumptions. We define the conjugacy $h$ 
separately on $U$ and $V$ by setting

$$
h(x):=\left\{\begin{array}{ll}
x & \text { for } x \in U \backslash\{0\} \\
e^{S \lambda^{-1} \ln \|x\|} x & \text { for } x \in V \backslash\{0\} \\
0 & \text { for } x=0
\end{array}\right\} .
$$

For arbitrary $x \in \mathbb{R}^{d}$ we define

$$
h(x):=h\left(x^{1}\right)+h\left(x^{2}\right),
$$

where $x=x^{1}+x^{2}$ is the unique decomposition of $x$ with $x^{1} \in U$ and $x^{2} \in V$. $h$ is invertible with

$$
h^{-1}(x)=g(x):=\left\{\begin{array}{ll}
x & \text { for } x \in U \backslash\{0\} \\
e^{-S \lambda^{-1} \ln \|x\|_{x}} & \text { for } x \in V \backslash\{0\} \\
0 & \text { for } x=0
\end{array}\right\} .
$$

and $g(x)=g\left(x^{1}\right)+g\left(x^{2}\right)$ for arbitrary $x \in \mathbb{R}^{d}$ : Note that

$$
\begin{aligned}
& h(g(x))=h\left(g\left(x^{1}\right)+g\left(x^{2}\right)\right)=h\left(g\left(x^{1}\right)\right)+h\left(g\left(x^{2}\right)\right), \\
& g(h(x))=g\left(h\left(x^{1}\right)+h\left(x^{2}\right)\right)=g\left(h\left(x^{1}\right)\right)+g\left(h\left(x^{2}\right)\right),
\end{aligned}
$$

since $h$ and $g$ respect the subspaces $U$ and $V$. Obviously, $h(g(x))=g(h(x))=x$ for all $x \in U$. For $x \in V \backslash\{0\}$ one obtains

$$
h(g(x))=h\left(e^{-S \lambda^{-1} \ln \|x\|} x\right)=e^{S \lambda^{-1} \ln \left\|e^{-S \lambda^{-1} \ln \|x\|} x\right\|} e^{-S \lambda^{-1} \ln \|x\|} x .
$$

Since $e^{-S \lambda^{-1} \ln \|x\|}$ is an orthogonal matrix, it follows

$$
h(g(x))=\left(e^{S \lambda^{-1} \ln \|x\|} e^{-S \lambda^{-1} \ln \|x\|}\right) x=x .
$$

Analogously one shows that $g(h(x))=x$. Continuity of $h$ and $h^{-1}$ follows since both maps preserve the Euclidean norm, i.e. $\|h(x)\|=\left\|h^{-1}(x)\right\|=\|x\|$ for all $x \in \mathbb{R}^{d}$ (Note that $U=V^{\perp}$ ). Thus, $h$ is a homeomorphism. The conjugacy identity can be checked separately on $U$ and $V$ : On $U=\operatorname{ker} S$ we have $S_{B} x \equiv S_{A} x$ and therefore

$$
h\left(e^{A t} x\right)=e^{A t} x=e^{(\lambda I+N) t} e^{S_{A} t} x=e^{(\lambda I+N) t} e^{S_{B} t} x=e^{B t} x=e^{B t} h(x) .
$$

By (5.8) we have $N S=0$. This implies $e^{N t} x=x$ for all $x \in V=\operatorname{im} S$, which yields

$$
\begin{aligned}
h\left(e^{A t} x\right) & =e^{\lambda^{-1} S \ln \left\|e^{\left(\lambda I+S_{A}\right) t} x\right\|} e^{\left(\lambda I+S_{A}\right) t} x=e^{\lambda^{-1} S \ln \left\|e^{\lambda t} x\right\|} e^{\lambda t} e^{S_{A} t} x \\
& =e^{\lambda t} e^{\lambda^{-1} S(\lambda t+\ln \|x\|)} e^{S_{A} t} x=e^{\lambda t} e^{\left(S+S_{A}\right) t} e^{\lambda^{-1} \ln \|x\|} x \\
& =e^{\lambda t} e^{S_{B} t} h(x)=e^{B t} h(x) .
\end{aligned}
$$

In order to show that $h$ is a Lipschitz conjugacy it suffices to show that $\left.h\right|_{V}$ is Lipschitz continuous. $\left.h\right|_{V}$ is obviously $C^{1}$ on $V \backslash\{0\}$. So we can prove Lipschitz continuity by giving an upper bound for $\left\|D h_{x}\right\|$. A straightforward calculation shows that

$$
D h_{x}=e^{S \lambda^{-1} \ln \|x\|}\left(I+S \lambda^{-1} \frac{x x^{T}}{\|x\|^{2}}\right) \text { for all } x \in V \backslash\{0\} .
$$

Since $S$ is skew-symmetric, we obtain

$$
\left\|D h_{x}\right\|=\left\|I+S \lambda^{-1} \frac{x x^{T}}{\|x\|^{2}}\right\| \leq\|I\|+|\lambda|^{-1}\|S\| \underbrace{\frac{\left\|x x^{T}\right\|}{\|x\|^{2}}}_{=1}=1+|\lambda|^{-1}\|S\| .
$$

The same argument can be used to show the Lipschitz continuity of $h^{-1}$. 
Now we consider the case of arbitrary - not necessarily hyperbolic - linear flows. For an arbitrary matrix $A \in \mathbb{R}^{d \times d}$ we have $\mathbb{R}^{d}=\mathbb{E}^{s}(A) \oplus \mathbb{E}^{u}(A) \oplus \mathbb{E}^{c}(A)$ and $A=A^{s} \oplus A^{u} \oplus A^{c}$ with $A^{\sigma}=\left.A\right|_{\mathbb{E}^{\sigma}(A)}$.

Corollary 5.7. For $A, B \in \mathbb{R}^{d \times d}$ the following are equivalent:

(i) $e^{A t} x$ and $e^{B t} x$ are Lipschitz conjugate.

(ii) $e^{\left(A^{s} \oplus A^{u}\right) t} x$ and $e^{\left(B^{s} \oplus B^{u}\right) t} x$ are Lipschitz conjugate and there exists $C \in \mathrm{Gl}(d, \mathbb{R})$ with $C A^{c} C^{-1}=B^{c}$.

Proof. Assuming the first statement, the second follows by $[\mathbf{2}, \S 1]$ and the same methods as in the proof of Corollary 5.4. For the converse, let $h: \mathbb{R}^{d} \rightarrow \mathbb{R}^{d}$ be the Lipschitz conjugacy from $e^{\left(A^{s} \oplus A^{u}\right) t} x$ to $e^{\left(B^{s} \oplus B^{u}\right) t} x$. Let $P$ be the projection onto $\mathbb{E}^{s}(A) \oplus \mathbb{E}^{u}(A)$ and $P^{c}$ be the projection onto $\mathbb{E}^{c}(A)$. Then $\tilde{h}(x):=h(P(x))+C^{-1} P^{c}(x)$ is a Lipschitz conjugacy from $e^{A t} x$ to $e^{B t} x$.

REMARK 3. The equation $N\left(S_{B}-S_{A}\right)=0$ says that the imaginary parts of the eigenvalues of $A$ and $B$ may only differ in the simple Jordan blocks. For example, the flows $e^{A_{1} t} x$ and $e^{A_{2} t} x$ corresponding to the system matrices

$$
A_{j}=\left[\begin{array}{cccccc}
\lambda & \mu_{j} & 1 & & & \\
-\mu_{j} & \lambda & & 1 & & \\
& & \lambda & \mu_{j} & & \\
& & -\mu_{j} & \lambda & & \\
& & & & \rho & \nu_{j} \\
& & & & -\nu_{j} & \rho
\end{array}\right] \quad(j=1,2)
$$

are Lipschitz conjugate if and only if $\mu_{1}=\mu_{2}$.

Acknowledgements. We would like to thank Fritz Colonius for numerous discussions.

\section{References}

1. C. Robinson, 'Dynamical Systems. Stability, Symbolic Dynamics, and Chaos', Boca Raton: CRC Press (1995).

2. N. H. Kuiper, 'The Topology of the Solutions of a Linear Differential Equation on $\mathbb{R}^{n}$, In: Hattori, A., ed., Manifolds-Tokyo 1973, pp. 195-203, Univ. Tokyo Press.

3. N. N. Ladis, 'The topological equivalence of linear flows', J. Differ. Equations 9 (1975) 938-947.

4. O. Perron, 'Über eine Matrixtransformation', Math. Z. 32 (1930) 465-473.

5. L. MARKus, 'Continuous matrices and the stability of differential systems', Math. Z. 62 (1955) 310-319.

6. B. Burago, Y. Burago, and S. Ivanov, 'A Course in Metric Geometry', Graduate Studies in Mathematics, Providence, Rhode Island: American Mathematical Society (2001).

7. D. Cohn, 'Measure Theory', Stuttgart: Birkhäuser (1980).

8. W. A. Coppel, 'Dichotomies in Stability Theory', Berlin: Springer-Verlag (1978).

\section{Kawan and T. Stender}

Institut für Mathematik

Universität Augsburg

Universitätsstrasse 14

86135 Augsburg

Germany

christoph.kawan@math.uni-augsburg.de torben.stender@math.uni-augsburg.de 